\title{
ARGOS testbed: study of multidisciplinary challenges of future spaceborne interferometric arrays
}

\author{
Soon-Jo Chung, MEMBER SPIE \\ David W. Miller, MEMBER SPIE \\ Olivier L. de Weck \\ Massachusetts Institute of Technology \\ Space Systems Laboratory \\ Cambridge, Massachusetts 02139 \\ E-mail: sjchung@mit.edu
}

\begin{abstract}
Future spaceborne interferometric arrays must meet stringent optical performance and tolerance requirements while exhibiting modularity and acceptable manufacture and integration cost levels. The Massachusetts Institute of Technology (MIT) Adaptive Reconnaissance Golay-3 Optical Satellite (ARGOS) is a wide-angle Fizeau interferometer spacecraft testbed designed to address these research challenges. Designing a space-based stellar interferometer, which requires tight tolerances on pointing and alignment for its apertures, presents unique multidisciplinary challenges in the areas of structural dynamics, controls, and multiaperture phasing active optics. In meeting these challenges, emphasis is placed on modularity in spacecraft subsystems and optics as a means of enabling expandability and upgradeability. A rigorous theory of beam-combining errors for sparse optical arrays is derived and flown down to the design of various subsystems. A detailed elaboration on the optics system and control system is presented based on the performance requirements and beam-combining error tolerances. The space environment is simulated by floating ARGOS on a frictionless airbearing that enables it to track both fast and slow moving targets. (๑) 2004 Society of Photo-Optical Instrumentation Engineers. [DOI: 10.1117/1.1779232]
\end{abstract}

Subject terms: synthetic apertures; apertures; space optics; imaging systems; optical systems.

Paper 030610 received Dec. 2, 2003; accepted for publication Mar. 5, 2004. This paper is a revision of a paper presented at the SPIE conference on Astronomical Telescopes and Instrumentation, Waikoloa, Hawaii, August 2002. The paper presented there appears (unrefereed) in SPIE Proceedings Vol. 4849.

\section{Introduction}

\subsection{Sparse Aperture Interferometric Arrays}

The quest for finer angular resolution in astronomy inevitably leads to larger aperture since the resolution is proportional to the wavelength over the diameter of a circular aperture. Unfortunately, the primary mirror diameter for space telescopes is limited by volume and mass constraints of current launch vehicles (ca. 4 to $5 \mathrm{~m}$ ) as well as the scaling laws of manufacturing costs. ${ }^{1}$ Since the cost of monolithic optics increases faster than their diameter squared, and mirrors such as the Hubble Space Telescope's are already at the edge of what is financially feasible, efforts are ongoing to break this trend by employing breakthrough technologies such as deployed segmented mirror telescopes and sparse aperture optics using interferometry. Whereas long baseline stellar Michelson ${ }^{2}$ interferometers feed light from independent collectors to a beam combiner to obtain interference fringes over a period of time (pupil plane beam combination), Fizeau ${ }^{2}$ interferometers produce a direct image with full instant $u-v$ coverage (image-plane beam combination). Hence, Fizeau interferometers are suitable for optical imaging of extended objects and rapidly changing targets. In contrast to the long baselines of Michelson interferometers, Fizeau interferometry systems tend to have compact telescope arrays. An optimal imaging con- figuration designed for sparse arrays was first proposed by Golay. ${ }^{3}$ Sparse arrays are promising for applications that do not require extremely high sensitivity (bright source present) and allow for a rather limited field of view ${ }^{4,5}$ (FOV). Diffraction-limited performance has been demonstrated for sparse or dilute-aperture telescopes with active phasing control. ${ }^{6}$ A notable project in the area of phased telescope arrays is the Multipurpose Multiple Telescope Testbed $^{7}$ (MMTT) built by the Air Force Research Laboratory (AFRL). The MMTT consists of four 20-cm-aperture telescopes phased together with a 15-arcmin FOV. The MMTT employs a complex laser interferometer metrology to sense wavefront error (WFE). The Multi-Aperture Imaging Array ${ }^{8}$ built by Lockheed Martin demonstrated phase diversity computation techniques for WFE sensing. This sparse array consists of afocal telescopes arranged in a $Y$ formation that are combined to a common focus in a Fizeau interferometer configuration. It demonstrated the first results of a broadband multiple-telescope imaging array phased over a significant FOV using an extended image projector in the lab. Research into WFE sensing and control has been extensively conducted for the Next Generation Space Telescope (renamed the James Webb Space Telescope). ${ }^{9}$

The Massachusetts Institute of Technology (MIT) Space Systems Laboratory (SSL) successfully completed the Mid- 


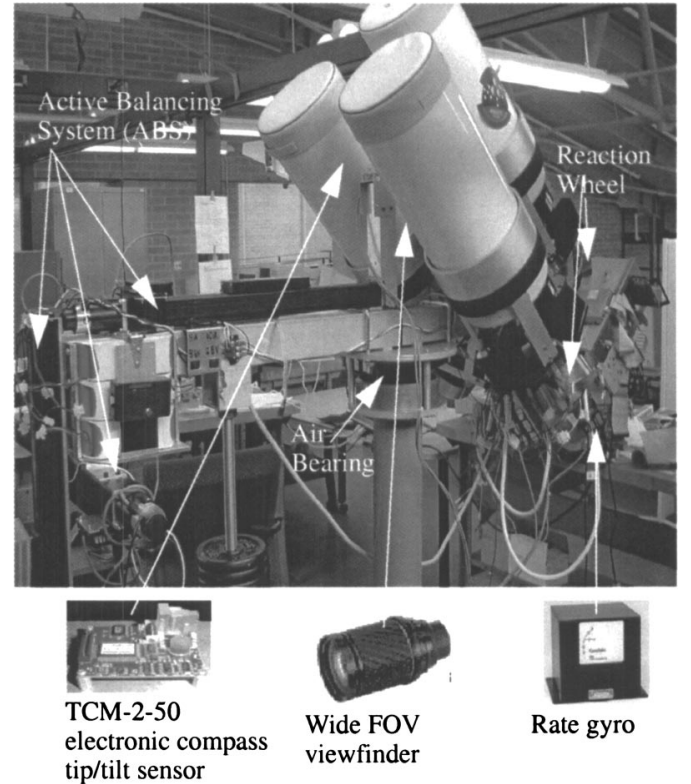

Fig. 1 Golay-3 ARGOS system with the three attitude control system (ACSs) shown at the bottom.

deck Active Control Experiment ${ }^{10}$ (MACE), a Space Shuttle flight experiment that flew on STS-67 in March 1995. The primary goal of the MACE project was to investigate approaches for achieving high-precision pointing and vibration control of future spacecraft. The MIT-SSL also developed The Origins Testbed ${ }^{11}$ (OT), a laboratory test article to capture the dynamics and control problems anticipated for future space telescopes. Built on the heritage of the MACE and the OT, a ground-based satellite testbed with a more sophisticated active optical payload system is presented in this paper. Most of the previous sparseaperture systems do not deal with real-world problems such as the vibrational coupling between a spacecraft structure and the wavefront errors propagating through the whole system. The presented testbed explores such issues while implemented on a nonrotationally constrained platform.

\subsection{Overview of ARGOS}

To better understand the technological difficulties involved in designing and building a sparse aperture array, the challenge of building a Golay-3 interferometer system was undertaken. The MIT Adaptive Reconnaissance Golay-3 Optical Satellite ${ }^{12}$ (ARGOS) exploits wide-angle Fizeau interferometer technology with an emphasis on modularity in the optics and spacecraft subsystems. The objective of the ARGOS project is to demonstrate the feasibility of a modular architecture for space-based optical systems.

To demonstrate a complete spacecraft in a 1- $g$ environment, the ARGOS system is mounted on a frictionless airbearing, as shown in Fig. 1, and has the ability to track fast orbiting satellites like the International Space Station (ISS) as well as slow moving objects such as point stars. The modular architecture emphasizes the use of replicated components and standard interfaces. The system consists of three identical apertures arranged in a Golay- 3 distribution. The light from these telescopes is combined in a center module and transmitted to a charge-coupled device (CCD).
Table 1 Key functional requirements.

\begin{tabular}{ll}
\hline \hline Key Requirements & \\
\hline Angular resolution & 0.35 arcsec at operating wavelength \\
Operating wavelength & $400-700 \mathrm{~nm}$ (visible) \\
FOV & $3 \mathrm{arcmin} \times 3 \mathrm{arcmin}$ \\
Field of regard (FOR) & $120 \mathrm{deg}$ \\
Signal-to-noise ratio (SNR) & 100 \\
ACS pointing accuracy & $\pm 1 \mathrm{arcmin}$ \\
Image acquisition time & 20 images $/ \mathrm{h}$ (max) \\
Autonomous operation time & up to $1 \mathrm{continuous} \mathrm{h}$ \\
\hline \hline
\end{tabular}

Wavefront sensing techniques are explored to mitigate initial misalignment and to feed back real-time aberrations into the optical control loop. The goal is to obtain an image of similar quality as the image received from a monolithic telescope using a single comparable aperture. The primary functional requirements of ARGOS are prescribed in Table 1.

The structure of this paper follows the step-by-step design procedures of ARGOS. A theory of interferometric beam combining errors is developed in the process and connected to the actual design and implementation of ARGOS. The paper also details the design of the optics based on the performance requirements and beam-combining tolerances. In addition, it includes the control avionics and strategies for a large-angle slewing and active interferometric beam combining.

\section{Physics of Sparse-Aperture Interferometric Arrays}

\subsection{Determination of Array Configuration}

Traditional image quality criteria such as resolution and encircled energy (EE) are inadequate for many sparseaperture or interferometric array applications. ${ }^{13}$ When looking at extended objects such as the Moon or faint distant nebulae, evaluation of an optical system involves more than simply looking at a point source response [point spread function (PSF)]. The modulation transfer function (MTF) is a better metric to evaluate the contrast (modulation) transfer characteristic of an extended object. Figure 2 shows the crucial relationship between the two most common image quality criteria-PSF and MTF. The PSF is the squared modulus of the Fourier transform of the complex pupil function. The optical transform function (OTF) is a Fourier transform of the PSF, and the MTF is an absolute value (magnitude) of the OTF. In Fig. 3, the PSF and MTF plots of a Golay-3-like array with $D=0.21 \mathrm{~m}$ (ARGOS subaperture diameter) and the array radius, $L=0.12 \mathrm{~m}$ (solid), 0.19 $\mathrm{m}$ (dash), $0.3 \mathrm{~m}$ (dot) are shown. A perfect monolithic aperture, free of optical aberrations, has a linearly decreasing MTF contrast characteristic (see the solid MTF line in Fig. 3 ). In case of a sparse array, the MTF suffers a contrast loss in the mid spatial frequency range, as shown in Fig. 3. We can observe that the MTF plot with $L=0.3 \mathrm{~m}$ exhibits two zero MTF values rather than one. The first zero denoted by $F_{r}$ is the practical spatial cutoff frequency, and defines the "practical resolution limit." The $F_{c}$ is the cutoff frequency, 


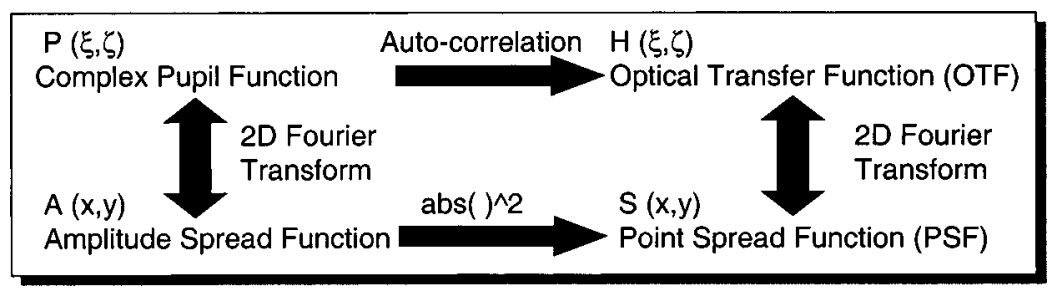

Fig. 2 The relationship between the PSF and OTF.

whose inverse indicates an angular resolution under the normal condition that there is no $F_{r}$ (another zero point) before $F_{c}$. Thus, the larger $F_{c}$ or $F_{r}$ is, the better is the angular resolution a sparse array will achieve.

In contrast with monolithic apertures, the method of full width at half maximum (FWHM) is not sufficient to determine the angular resolution. Assuming that angular resolution is fully determined by the array size, the PSF can reveal the highest achievable angular resolution. This assumption holds especially for very large baseline Michelson interferometers. Figure 3 shows that the width of the mainlobe of the PSF plot is getting smaller, indicating improving angular resolution as we increase the array size $L$. The Fizeau interferometer, however, requires an instantaneous full $u-v$ coverage, which limits the practical resolution. In addition, as discussed, considerable contrast loss in the mid-spatial-frequency range should be avoided. The angular resolution from the MTF plot of a Golay-3 type sparse imaging array is approximated as ${ }^{12}$

$\theta_{r}=1.22 \frac{\lambda}{D_{\mathrm{eff}}}=2.44 \frac{\lambda}{3 L+\left(4 D^{2}-3 L^{2}\right)^{1 / 2}}$.
As $L$ is increased, the array becomes sparser, which, as a result, boosts the heights of sidelobes in the PSF. The MTF plot at $L=0.3 \mathrm{~m}$ (dotted line in Fig. 3) has two zeros while others have only one. In that case, the resolution is limited not by $F_{c}$ but by $F_{r}$, whose inverse is the angular resolution of a single aperture $(1.22 \lambda / D)$. Thus, in that case, the sparse array has no advantage over a single subtelescope except for increased light-gathering power. The array configuration of ARGOS is selected as $L=0.19185 \mathrm{~m}$ for $D$ $=0.21 \mathrm{~m}$ subtelescopes. This results in a better theoretical angular resolution of 0.35 arcsec for the 550-nm wavelength rather than 0.55 arcsec of a single aperture, compromising with a reasonable MTF characteristic.

\subsection{Beam Combining Errors}

There are three major wavefront errors that must be controlled at the beam combiner to achieve phased beam combining. Those errors are optical path difference (OPD), tip/ tilt error, and pupil-mapping error.
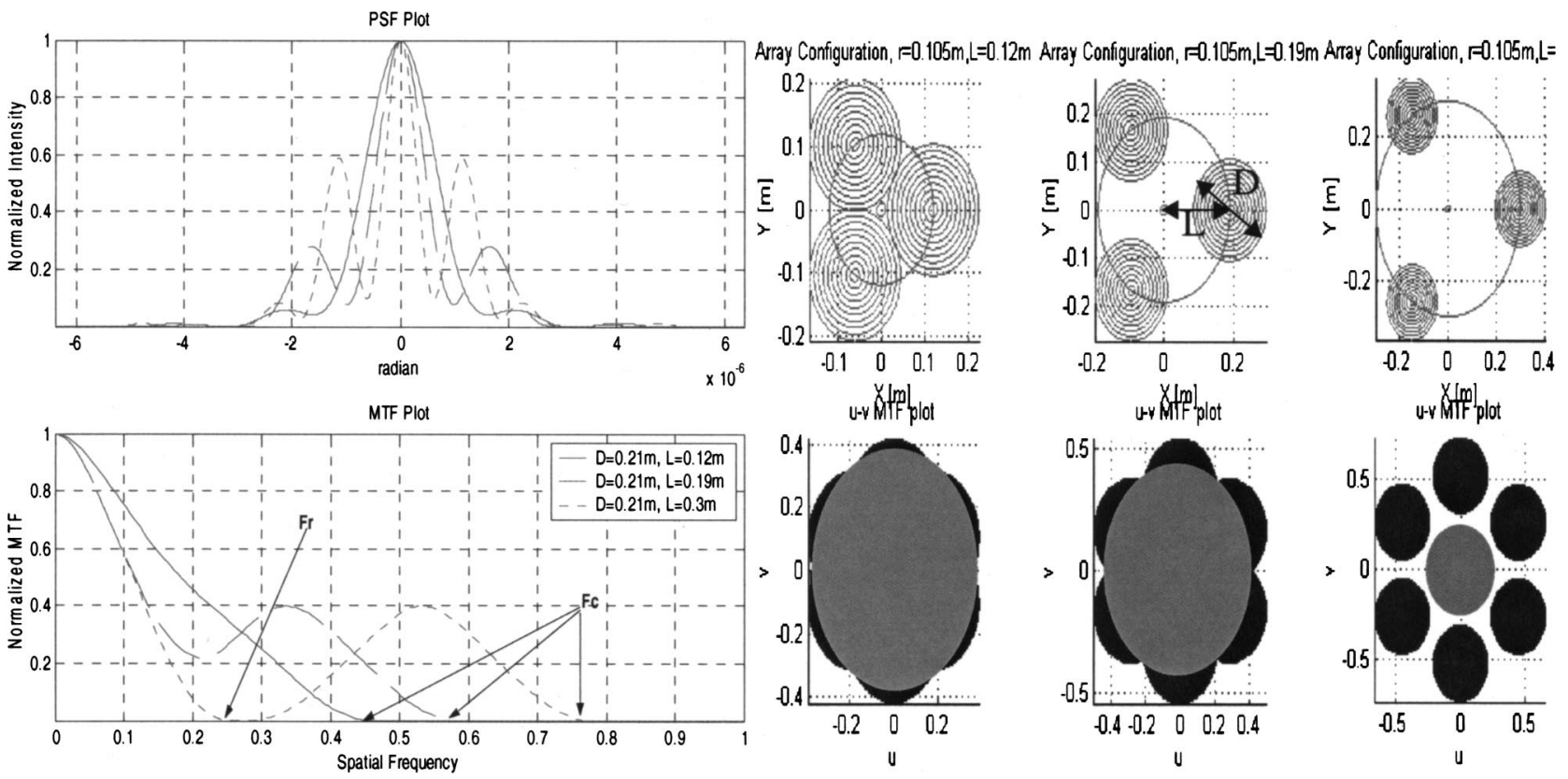

Fig. 3 PSF and MTF plots when $D=0.21 \mathrm{~m}$ and $L=0.12 \mathrm{~m}$ (solid), $0.19 \mathrm{~m}$ (dash), and $0.3 \mathrm{~m}$ (dot). The corresponding array configurations are shown to the right of PSF-MTF plot. The black-gray figure is an MTF plot. Gray circles indicate the practical cutoff frequency. 


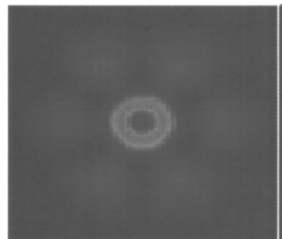

(a)

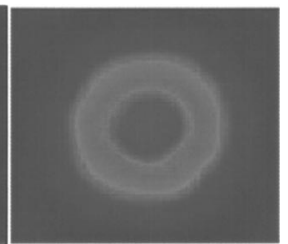

(c)
Fig. 4 PSF plot of Golay-3 array with (a) 0 OPD, (b) $0.5 \lambda$ OPD, and (c) $1.0 \lambda$ OPD.

\subsubsection{OPD (piston) error}

We can plot the effects of OPD errors using the interferometry equation given by Mennesson and Mariotti ${ }^{14}$

$$
\begin{aligned}
I \propto \mid & \left|\frac{\pi D[1+\cos (r)]}{\lambda}\right|^{2}\left|\frac{J_{1}(\pi D \sin r / \lambda)}{\pi D \sin r / \lambda}\right|^{2} \\
& \times\left|\sum_{k=1}^{n} \exp \left[j 2 \pi\left(L_{k} r / \lambda\right) \cos \left(\delta_{k}-\theta\right)\right] e^{j \phi_{k}}\right|^{2},
\end{aligned}
$$

where $r$ is the off-axis angular direction, $\theta$ is the azimuth angle, $D$ is a subaperture diameter, $\left(L_{k}, \delta_{k}\right)$ are the polar coordinates of the array configuration, and $\phi_{k}$ is the phase shift.

Numerical simulations using Eq. (2) were performed to investigate the effects of OPD errors, as shown in Fig. 4. As the piston error increases, two major deviations develop over the envelope of the PSF. First, the main envelope shifts in the direction of the piston error. The resultant direction of the envelope shift is the vector sum of piston error directions weighted by the amount of error. Second, the peak intensity is reduced compared to the nominal PSF without any piston errors resulting in a reduced Strehl ratio (SR). The size of the mainlobe also expands showing a degraded angular resolution [see Fig. 4(c)]. When the piston error is $0.1 \lambda$ between apertures, the peak intensity is $98 \%$ of the normal intensity. The beam combining piston error tolerance is chosen to be $0.1 \lambda=55 \mathrm{~nm}$ for most of interferometric beam combining applications.

\subsubsection{Tip/tilt error}

The approach employed here to analyze tilt errors is to further segment each aperture into smaller elements for analysis (finite element method). The PSF is calculated by summing up the interference from each element. The phase difference at the central point due to tilt errors are added to the interference term of Eq. (2):

$$
\begin{aligned}
I \propto \mid & \left|\frac{\pi d[1+\cos (r)]}{\lambda}\right|^{2}\left|\frac{J_{1}(\pi d \sin r / \lambda)}{\pi d \sin r / \lambda}\right|^{2} \\
& \times \mid \sum_{k=1}^{m} \exp \left[j 2 \pi\left(L_{k} r / \lambda\right) \cos \left(\delta_{k}-\theta\right)\right] \\
& \times\left.\exp \left[j d_{k} \sin \left(\mathrm{tilt}_{n}\right) 2 \pi / \lambda\right]\right|^{2},
\end{aligned}
$$

where $D$ in Eq. (2) is replaced with the smaller element diameter $d, d_{k}$ is the distance from the aperture center to the $k$ ' th element center, tilt ${ }_{n}$ denotes the tilt in radians for the $n$ ' th aperture, $k$ ranges from 1 to $m$, and $m$ equals the number of apertures $(n)$ multiplied by the number of smaller elements of each aperture. By reading the SR values from the PSF plots under the influence of tilt errors, the maximum allowable tilt error at the beam-combining stage is determined. This numerical method predicts that the tilt tolerance between each beam entering the beam combiner should be less than $20 \mu \mathrm{deg}(0.072 \operatorname{arcsec}=0.35 \mu \mathrm{rad})$.

\subsubsection{Pupil mapping error}

If coherent imaging is to be achieved over any significant FOV, the pupil mapping process must be performed such that the exit pupil is an exact (scaled) replica of the entrance pupil. This constraint is commonly called the "golden rule" of beam combining. From the geometry of Fig. 5, Faucherre et al. ${ }^{5}$ derived the net OPD error due to the incorrect pupil mapping,

$$
\mathrm{OPD}_{\text {net }}=|B \sin \alpha-b \sin \beta|=\left|B \sin \alpha-\frac{B}{m_{s}} \sin \left(m_{a} \alpha\right)\right| \text {, }
$$

where $m_{a}$ is the aperture magnification factor $(D / d), m_{s}$ is the baseline magnification factor $(B / b), \alpha$ is half FOV of the target, and $\beta$ is magnified field angle through the subtelescope array.
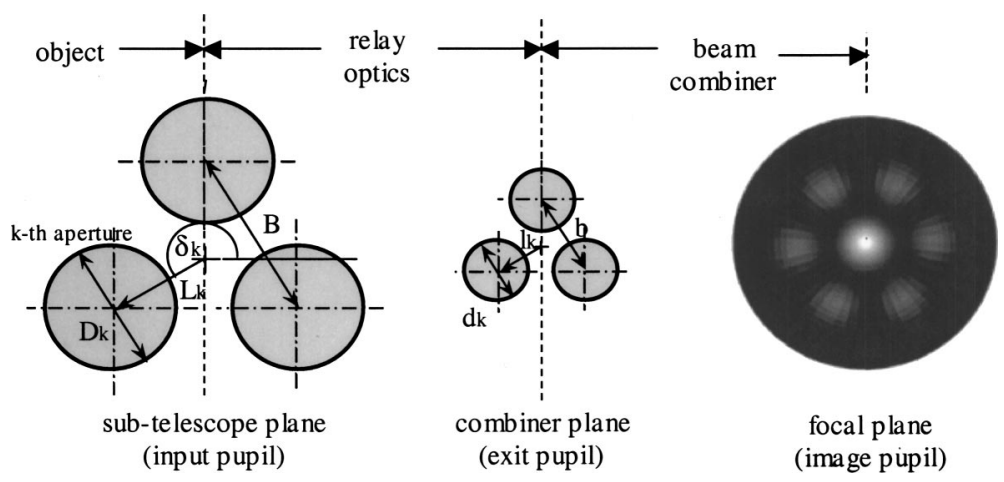

Fig. 5 The golden rule of beam combining, pupil mapping. 
According to the discussion in Sec. 2.2.1, 1/10 $\lambda$ phasing is desired to meet the SR and angular resolution requirements. Thus, equating Eq. (4) with $1 / 10 \lambda$ and small angle approximation $\sin \alpha \cong \alpha$ results in

$B \alpha\left|1-\frac{m_{a}}{m_{s}}\right|=\frac{\lambda}{10}$

A calculation of shear error tolerance is performed with an assumption that there is no magnification error (subtelescopes are strictly afocal with exactly the same magnification). Then, the correct magnification is $m_{a}=D / d=B /(b$ $+\Delta b)$. The incorrect mapping $m_{s}$ is still $B / b$. Inserting $m_{a}$ and $m_{s}$ into Eq. (5) results in

$\alpha\left|B-b m_{a}\right|=b \alpha\left|\frac{B}{b}-\frac{B}{b+\Delta b}\right|=\alpha \frac{B \Delta b}{b+\Delta b}=\frac{\lambda}{10}$.

Solving for $\Delta b$, gives

$\Delta b=\frac{\lambda}{10 m_{a} \alpha}$.

Equation (7) means that the allowable shear error is inversely proportional to both aperture magnification $m_{a}$ and off-axis target angle $\alpha(\mathrm{FOV} / 2)$. However, the assumption of no magnification error used for Eq. (7) is inadequate since perfectly replicating any subaperture is impossible in terms of manufacturability. There usually exists some performance window specified by optics manufacturers. The objective of this calculation is to find out how precise the magnification tolerance level must be in order to achieve coherent beam combining. If the magnification tolerance is too stringent to be manufactured, methods of relaxing this tolerance will be explored.

For both incorrect $m_{a}(=D / d)$ and incorrect $m_{s}$ $(=B / b)$, the acceptable magnification ratios are defined as follows:

$m_{a}^{\text {correct }}=m_{a}+\Delta m_{a}=\frac{B}{b+\Delta b}=m_{s}^{\text {correct }}$.

Inserting these results into Eq. (5), gives

$$
\begin{aligned}
\alpha b\left|\frac{B}{b}-m_{a}\right| & =\alpha b\left|\frac{B}{b}-\frac{B}{b+\Delta b}+\Delta m_{a}\right| \\
& =\alpha\left|\frac{\Delta b B}{b+\Delta b}+\Delta m_{a} b\right|=\frac{\lambda}{10} .
\end{aligned}
$$

We can remove $b$ by using Eq. (8):

$\alpha\left|\Delta b m_{a}^{\text {correct }}+\Delta m_{a}\left(\frac{B}{m_{a}^{\text {correct }}}-\Delta b\right)\right|=\frac{\lambda}{10}$.

Setting $\alpha=\mathrm{FOV} / 2, m_{a}^{\text {correct }}=m$ and $B=\sqrt{3} L$ and for a Golay-3 array leads to

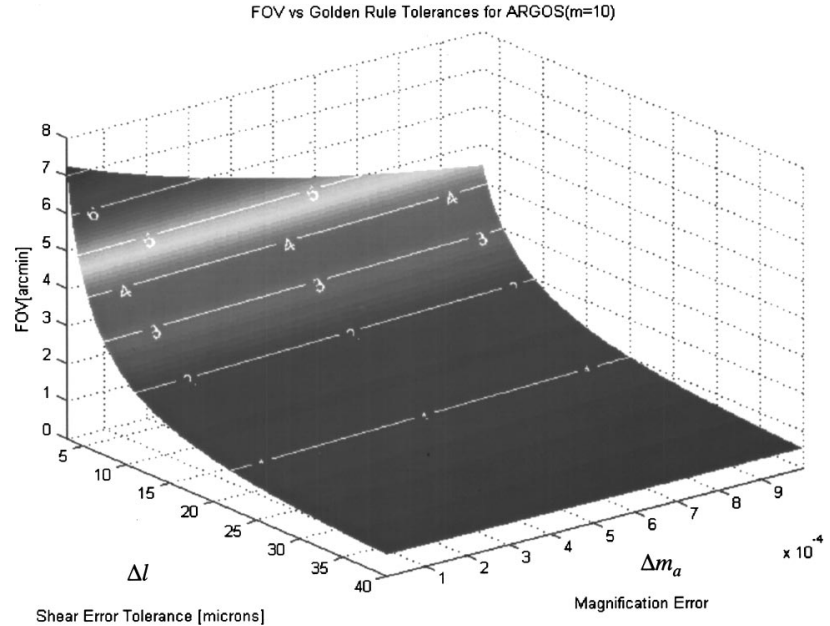

Fig. 6 FOV versus pupil mapping tolerances for ARGOS with achievable magnification tolerance.

$\mathrm{FOV}\left|m \Delta l+\frac{\Delta m L}{m}-\Delta m \Delta l\right|=\frac{\lambda}{5 \sqrt{3}}$.

From Eq. (11), we can find the FOV as a function of magnification $(m)$ and pupil mapping tolerances $(\Delta l$ and $\Delta m)$ :

$\mathrm{FOV}=\frac{\lambda}{5 \sqrt{3}|m \Delta l+(\Delta m L / m)-\Delta m \Delta l|}$.

We can also represent the shear error tolerance $\Delta l$ as a function of FOV, magnification $(m)$ and magnification tolerance $(\Delta m)$ for a positive $\Delta m$ :

$\Delta l=\frac{\lambda}{5 \sqrt{3} \mathrm{FOV}(m-\Delta m)}-\frac{\Delta m L}{m^{2}-m \Delta m}$.

\subsubsection{Pupil mapping error for ARGOS}

These results are graphically plotted in Figs. 6 and 7. At the preliminary design phase of ARGOS, $12 \mu \mathrm{m}$ was suggested for the shear error tolerance from Eq. (7) with an assumption of no magnification error $(\mathrm{FOV}=4 \mathrm{arcmin}$, magnification $=10)$. However, 12- $\mu \mathrm{m}$ shear error cannot produce the FOV requirement of ARGOS (3 arcmin) for any range of magnification error in Fig. 6. We can tighten shear error tolerance to meet the FOV requirement or we can relax the FOV requirement by shrinking the region of interest within the whole FOV. Therefore, the pupil mapping process is the primary limiting factor deciding the reasonable FOV of a sparse aperture interferometric array. ARGOS's subtelescope collimators are designed with a tolerance of \pm 0.0095 with a nominal magnification factor of 10. However, the ARGOS subtelescopes have a focusing knob that can control the distance between the primary mirror and the secondary mirror, thereby controlling the size of the beam more precisely. It is usually considered that a $1 / 1000$ magnification tolerance requirement is too expensive to manufacture. Figure 7 describes how the magnification of a subaperture affects the shearing tolerance of a 


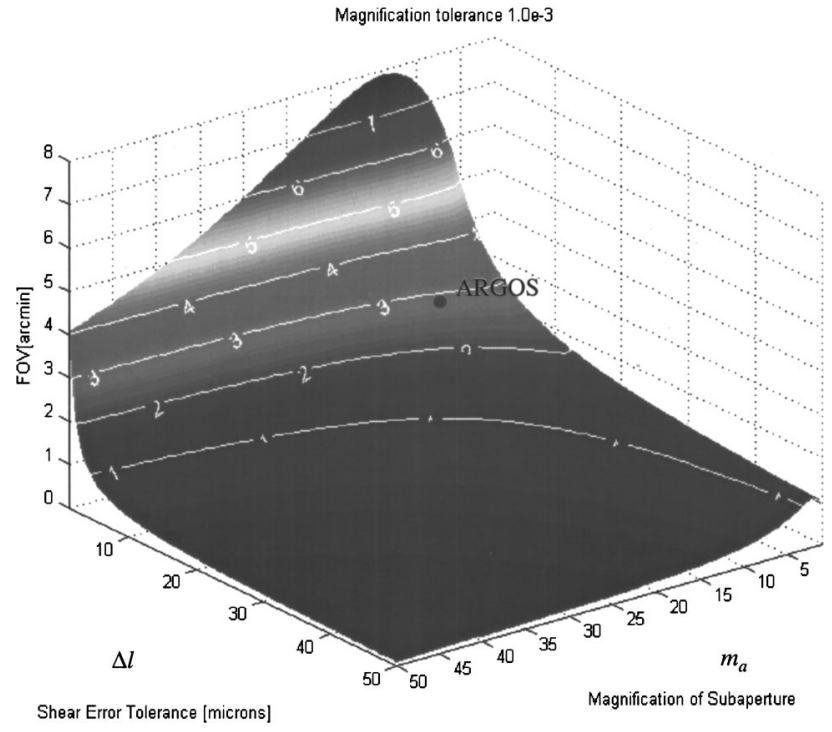

Fig. 7 Magnification versus shear tolerance with magnification error $=0.001$

sparse aperture imaging system. This plot implies that subaperture magnification can be tuned to maximize allowable shear error $\Delta l$ (lateral pupil mapping error) thereby reducing control complexity. For a magnification of 10, we can increase the shear tolerance value sufficiently high at the expense of the reduced FOV.

\subsection{Optics Design and Implementation}

\subsubsection{Subaperture}

The overall cost of the ARGOS optics system could be significantly reduced by selecting one of the highest precision optics commercially off-the-shelf (COTS) telescope. However, it was necessary to customize a collimating lens to convert the Dall-Kirkham-type focal telescope to an afocal telescope with a magnification ratio of 10 . The collimating lens is placed into the baffle of the telescope to make the system compact. A trade-off analysis was performed on several different cemented doublets that were optimized extensively by ZEMAX. One drawback of the cemented doublet is that it has bonded glasses, therefore if there is a change of temperature, the doublet may fail. Although a doublet with $\mathrm{CaF}_{2}$ performs best in reducing chromatic aberrations, the high coefficients of thermal expansion (CTEs) of $\mathrm{CaF}_{2}$ (18.3) forced to find another glass combination for efficient achromatic doublet design. Smith ${ }^{15}$ suggests FK51 (as a crown element) with a KzFS or LaK glass (as a flint). Although the maximum focal shift range can be minimized to $247 \mu \mathrm{m}$ with FK51-KzFS11, it was not the best choice due to the residual aberrations [root mean square (rms) wavefront errors predicted by ZEMAX]. The final FK51BaK2 design achieves $271.6 \mu \mathrm{m}$ chromatic focal shift range.

\subsubsection{Design of OPD and tilt/tip controller}

When strictly looking at two designs of optical delay lines (ODL) in Fig. 8, a perpendicular design seems to offer the most benefits. For the perpendicular design, a multiaxis fast

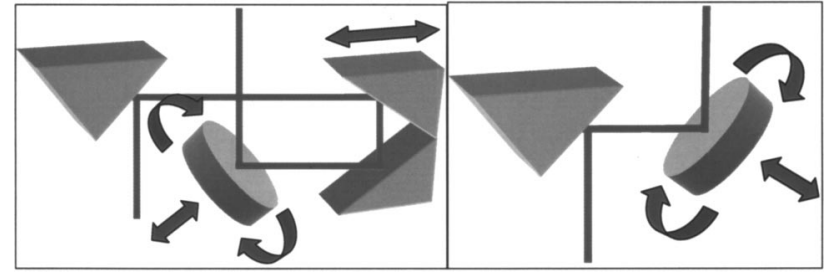

Fig. 8 Perpendicular ODL design with FSM (left) and parallel ODL design coupled with FSM (right).

steering mirror (FSM) could be used to generate the required tip/tilt actuations as well as fine optical path difference control. FSMs have very fine resolutions so depending on them for fine OPD control will enable us to have a more reasonable coarse control for the ODL. When the parallel ODL design is coupled with a FSM (Fig. 8), the resulting design is very simple and more cost-effective than the perpendicular design. This design cannot perform coarse OPD control, but this design is more compact resulting in easier integration with the structural design. In addition, there is no need for a translational stage or rooftop mirror, which reduces the cost and control complexity, and there is also a greater total reflectance since there are fewer mirrored surfaces. Fewer mirrored surfaces also lead to fewer structural misalignment errors. In both the perpendicular ODL design and the parallel ODL design, fine OPD control is coupled with shear control so that $\Delta$ Fine $\mathrm{OPD}=\Delta$ Shear. The effect that a change in fine OPD would have on shear, is not very significant and could be ignored for adjustments $<10 \mu \mathrm{m}$. This is because we have a much tighter tolerance on piston error $(50 \mathrm{~nm})$ than the $12-\mu \mathrm{m}$ shear error. A fine resolution multiaxis FSM, capable of controlling the tilt/tip as well as piston motion, was selected as the ODL-FSM actuator of ARGOS. The FSM has to be able to compensate for any errors in its mounting. Therefore a high-precision mount with a range up to $7 \mathrm{deg}$ was selected for the FSM actuators (see Fig. 10 and Table 2 in Sec. 2.3.3).

\subsubsection{Pyramidal mirror and beam combiner}

The pyramidal mirror turns all three beams $45 \mathrm{deg}$ into the beam combiner. A custom pyramidal mirror is chosen due to the cost of making one out of regular mirrors. The main reason for the high cost is thin mirrors that cost $\$ 1000+$, and then mount them to an accuracy of $\pm 0.001 \mathrm{deg}$ $( \pm 3.6 \mathrm{arcsec})$. The pyramid cannot be made from the regular thickness mirrors since they constrain the beam diameter. We customized a pyramidal mirror with a surface accuracy of $\lambda / 10$ peak to valley, \pm 3 arcsec angle error, and a $50-\mathrm{mm}$ clear aperture. The substrate material is BK7 with a coating of AlSiO (aluminum with silicon monoxide). The reflectance will be approximately $90 \%$ in the visible range. The two PSFs shown in Fig. 9 demonstrate how the FSM can compensate for the \pm 3 arcsec errors in the pyramid. To compensate for pyramid errors the FSM has to align itself so that the two reflecting surfaces are parallel. The pyramidal mount is composed of two stages. The first stage provides all of the angular adjustments and the second handles $X$ and $Y$ translation in the entrance pupil of the beam combiner. The $X-Y$ translation stage is small enough to fit behind the tip/tilt rotation stage and has the load capacity to 

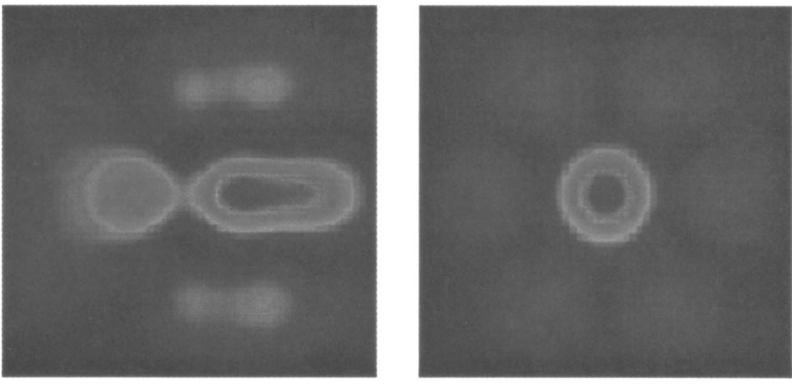

Fig. 9 PSF simulation of ARGOS with 3-arcsec tilt error of the pyramidal mirror $(S R=0.444$, left). The PSF with FSM correction ( $S R=0.960$, right).

hold both the second stage and the mirror. Any additional $Z$ directional error can be offset by the FSM mounts. The specifications of the ARGOS optical actuators and mounts are tabulated in Table 2 and their pictures are shown in Fig. 10.

Two options available for the image-plane beam combiner are either reflecting or refracting optics. The reflecting beam combiner is compact when compared to a refractor. Unfortunately, the secondary mirror of a Cassegrain telescope would partially block the three incoming beams in any possible configurations to obey the golden rule discussed in Sec. 2.2.3. A single parabolic mirror was considered, however, there was not enough space between the pyramidal mirror. Had we used reflecting optics, there would be nothing available as COTS, therefore it would have to be custom manufactured increasing cost significantly. A reflector would also complicate the relay optics significantly since we would not be able to use the pyramidal mirror. In contrast, a refracting combining telescope has many advantages. It allows for very simple relay optics. It is available as COTS with high-quality optics such as Takahashi FSQ-106N (see Fig. 10). This telescope has significantly less chromatic aberration than other COTS telescopes. This is due to its four-element design, two of which are fluorite. It has a diameter of $106 \mathrm{~mm}$, and a 530-mm focal length, resulting in a total system focal length of 5300 $\mathrm{mm}$.

\subsubsection{CCD system design}

The optics performance requirements driving the CCD camera selection are SNR and FOV. It was pointed out that the pupil mapping process is the primary limiting factor deciding the reasonable FOV of a sparse aperture interferometric array (see Sec. 2.2). This means that the pupil mapping tolerances become expensive as we increase the FOV.

Table 2 Optical actuators and mounts specifications.

\begin{tabular}{ccccc}
\hline \hline Model & Angular Range & $\begin{array}{c}\text { Angular } \\
\text { Resolution }\end{array}$ & $\begin{array}{c}\text { Linear } \\
\text { Range }\end{array}$ & $\begin{array}{c}\text { Linear } \\
\text { Resolution }\end{array}$ \\
\hline FSM & $\pm 600 \mu \mathrm{rad}$ & $\begin{array}{c} \pm 0.05 \mu \mathrm{rad} \\
\pm 0.0008 \mathrm{deg}\end{array}$ & $12 \mu \mathrm{m}$ & $0.2 \mathrm{~nm}$ \\
FSM mount & $\pm 7 \mathrm{deg}$ & $\begin{array}{c} \pm 0.0 \mathrm{~m} \\
( \pm 14 \mu \mathrm{rad})\end{array}$ \\
$\begin{array}{c}\text { Pyramid mirror } \\
\text { mount }\end{array}$ & $\pm 4 \mathrm{deg}$ & $\begin{array}{c} \pm 2 \mathrm{arcsec} \\
( \pm 9.6 \mu \mathrm{rad})\end{array}$ & $13 \mathrm{~mm}$ & $3 \mu \mathrm{m}$ \\
\hline \hline
\end{tabular}

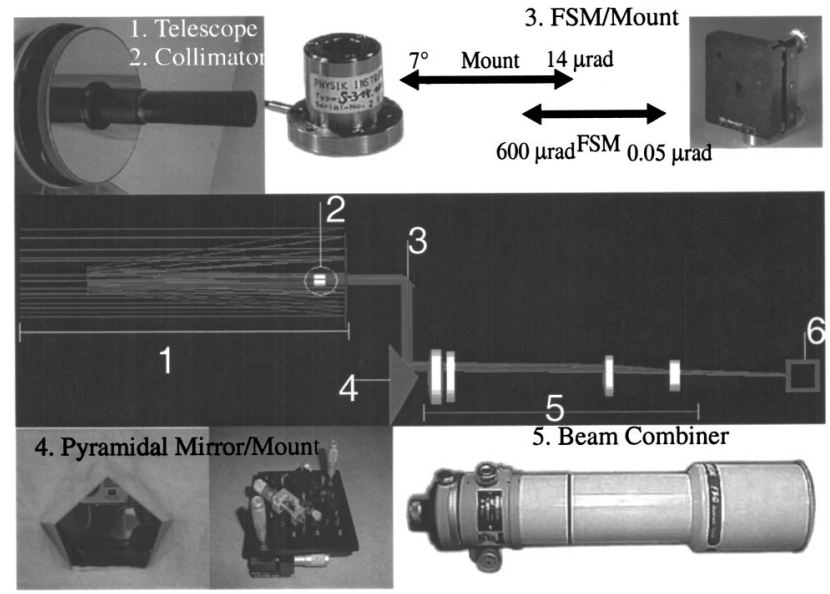

Fig. 10 Pictures of optical components.

In addition, the off-axis imaging aberrations such as distortion and field curvature become dominant as we increase the FOV. Therefore, the CCD system of a sparse aperture imaging system should be carefully designed to meet the optics performance requirements while maintaining the reasonable FOV large enough to capture the targets of interest. The following relationship is used to find an appropriate CCD pixel size and the number of CCD pixels:

$\mathrm{FOV}=\frac{d}{f} n=2.44 \frac{\lambda}{D_{\mathrm{eff}}} Q n$,

where $n$ is the number of pixels along the $x$ axis or the $y$ axis of a CCD matrix, $d$ is the pixel size, $f$ is the system effective focal length, and $Q$ is a quality factor. The $\mathrm{Ny}$ quist sampling criterion tells us that two pixels are generally required to properly record a star's image $(Q=0.5)$.

A sparse aperture can cause a substantial depression in the MTF of the system, resulting in a low-contrast and unsatisfactory SNR image. This can be compensated by image reconstruction, such as by using a Wiener-Helstron filter, but at the expense of greater noise sensitivity compared to a monolithic aperture. ${ }^{16}$ Images acquired by modern CCD cameras may be contaminated by a variety of noise sources. Three primary noise sources are discussed in this section: photon noise, read-out noise, dark current.

When the physical signal detected by a CCD is based on light, the quantum nature of light plays a significant role. Photon noise is derived by calculating the number of photons received through an imaging system, and is therefore not independent of light source. The spectral energy distribution of a blackbody is given by Planck's law: ${ }^{17}$

$E(\lambda)=\frac{2 \pi h c^{2}}{\lambda^{5}} \frac{1}{\exp (c h / k T \lambda)-1}$,

where $E(\lambda)$ is the energy per unit wavelength (also called the spectral irradiance measured in $\left.\mathrm{Wm}^{-2} \mu \mathrm{m}^{-1}\right), \lambda$ is the wavelength, $h$ is Planck's constant (6.6260755 $\times 10^{-34} \mathrm{~W} \mathrm{~s}^{2}$ ), $T$ is the absolute temperature of a target, $c$ is the speed of light, and $k$ is Boltzmann's constant $\left(1.380658 \times 10^{-23} \mathrm{~W} \mathrm{~s} / \mathrm{K}\right)$. 
Then the input power at the entrance to a sparse aperture array is calculated by

$P_{\text {in }}=\int_{\lambda_{1}}^{\lambda_{2}} E(\lambda) \mathrm{d} \lambda\left(\frac{D}{4}\right)^{2}\left(\frac{1.22 \lambda}{D_{\text {eff }}}\right)^{2} N$,

where the integration term integrates $E(\lambda)$ from Eq. (15) over the operating wavelengths, $N$ is the number of subapertures, $D$ is the diameter of a subaperture, and $D_{\text {eff }}$ is the effective diameter of a sparse aperture array.

Using Eq. (16), the number of available photons can be determined as follows:

$N_{p}=P_{\text {in }} \tau_{0} T_{i} \frac{\lambda}{h c}$,

where $T_{i}$ is the integration time, $\tau_{0}$ is the optical transmission factor (typically 0.75 ), $c$ is the speed of light, and $h$ is the Planck constant defined in Eq. (15).

The number of signal electrons is computed by multiplying $N_{p}$ with the CCD quantum efficiency:

$$
\begin{aligned}
N_{\text {signal }} & =P_{\text {in }}\left(\tau_{0} \frac{\lambda}{h c} \mathrm{QE}\right) T_{i} \\
& =\int_{\lambda_{1}}^{\lambda_{2}} E(\lambda) \mathrm{d} \lambda\left(N \frac{D^{2}}{D_{\text {eff }}^{2}}\right)\left(\frac{1.22 \lambda}{4}\right)^{2}\left(\tau_{0} \frac{\lambda}{h c} \mathrm{QE}\right) T_{i},
\end{aligned}
$$

where $\mathrm{QE}$ is the quantum efficiency of a CCD. The term $N\left(D^{2} / D_{\text {eff }}^{2}\right)$ is commonly called the filling factor of an interferometric array.

The probability distribution for the average number of photons received by a CCD is known to be Poisson. The standard deviation of the Poisson distribution is the square root of the average value. Accordingly, the total number of photon noise electrons is

$$
\begin{aligned}
N_{\mathrm{PN}} & =\sqrt{N_{\text {signal }}} \\
& =\left[\int_{\lambda_{1}}^{\lambda_{2}} E(\lambda) \mathrm{d} \lambda\left(N \frac{D^{2}}{D_{\text {eff }}^{2}}\right)\left(\frac{1.22 \lambda}{4}\right)^{2}\left(\tau_{0} \frac{\lambda}{h c} \mathrm{QE}\right) T_{i}\right]^{1 / 2} .
\end{aligned}
$$

By cooling the CCD chip it is possible to efficiently reduce the number of thermal electrons that produce thermal noise or dark current. As the integration time increases, the number of thermal electrons increases:

$N_{\mathrm{DC}}=R_{\mathrm{DC}} T_{i}$,

where $R_{\mathrm{DC}}$ is the dark current rate and $T_{i}$ is the integration time.

Readout noise (on-chip electronic noise) originates in the process of reading the signal from the sensor through the field effect transistor (FET) of a CCD chip. This noise can become a significant component in the overall SNR at very low signal levels.

Finally, we can calculate the SNR of a sparse aperture array based on the noise characteristics of a CCD system, as already discussed. In general, photon noise, dark current noise, and readout noise are considered as dominant noise contributors:

$\mathrm{SNR}=\frac{N_{\text {signal }}}{\left(N_{\mathrm{PN}}^{2}+N_{\mathrm{DC}}+N_{R}^{2}\right)^{1 / 2}}$,

where $N_{\text {signal }}$ is the number of signal electrons in Eq. (18), $N_{\text {PN }}$ is the number of photon noise electrons in Eq. (19), $N_{\text {DC }}$ is the number dark current noise electrons in Eq. (20), and $N_{R}$ is the number of readout noise electrons specified by a manufacturer.

The SNR can be defined again expanding all the equations for noise electrons:

$\mathrm{SNR}=\frac{\int_{\lambda_{1}}^{\lambda_{2}} E(\lambda) \mathrm{d} \lambda\left[N\left(D^{2} / D_{\text {eff }}^{2}\right)\right](1.22 \lambda / 4)^{2}\left[\tau_{0}(\lambda / h c) \mathrm{QE}\right] T_{i}}{\left\{\int_{\lambda_{1}}^{\lambda_{2}} E(\lambda) \mathrm{d} \lambda\left[N\left(D^{2} / D_{\text {eff }}^{2}\right)\right](1.22 \lambda / 4)^{2}\left[\tau_{0}(\lambda / h c) \mathrm{QE}\right] T_{i}+\left(R_{\mathrm{DC}} T_{i}\right)+N_{R}^{2}\right\}^{1 / 2}}$.

Using Eq. (22), the optimal frame rates and sensitivities can be obtained to achieve the required SNR for a given target.

\subsubsection{Structural misalignment tolerancing}

Using the mode of nonsequential ray tracing of ZEMAX, a complete ARGOS optics layout is constructed based on the optical specifications of a subaperture, pyramidal mirror, and the beam combining telescope, as shown in Fig. 11. We intentionally perturb the subtelescope or pyramidal mirror to determine allowable structural misalignment, and we compensate the tilt error by changing the tilt angle of the fold mirror attached to the FSM. At 0.01-deg tilt of a subaperture, a pure FSM motion cannot restore the SR above 0.8 . But the addition of FSM piston motion can restore the SR value to 0.859 . We could achieve a SR of 0.859 (which is above diffraction limited) over a 0.01-deg tilt. But due to a magnification factor 10, the FSM compensation exceeded its max range $(0.6 \mathrm{mrad}=0.034 \mathrm{deg})$. Since we mounted a FSM onto a precision tip-tip mount, which is capable of 


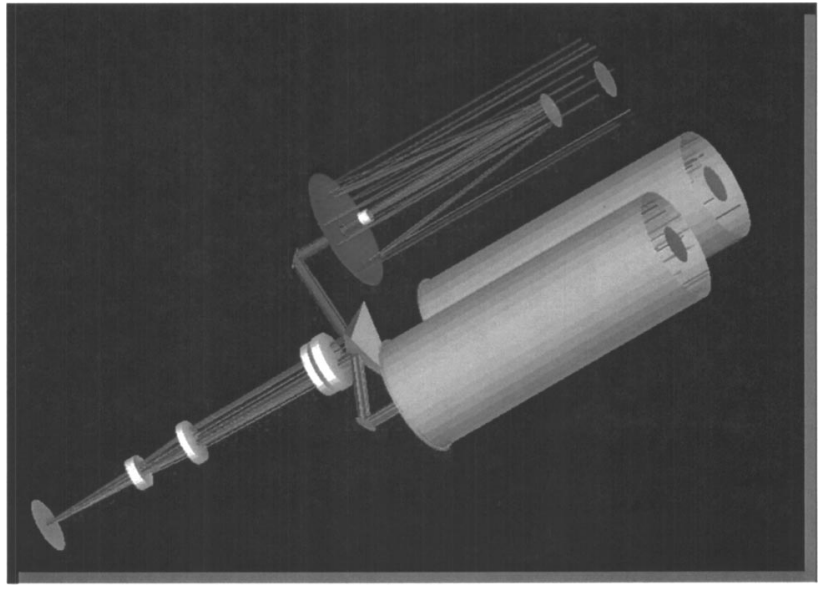

Fig. 11 ZEMAX 3-D nonsequential ray tracing.

several arcsecond adjustment (see Table 2), this static error does not limit the FSM performance. However, it is much safer to have a FSM within a range of eliminating a possible maximum alignment error, $0.005 \mathrm{deg}$ or 15 arcsec for subtelescope structural misalignment is suggested.

By assuming that all other optical components are perfectly aligned and the FSM can compensate for all the residual tilt errors, the tilt errors for each surface of the pyramidal mirror are calculated. When the tilt error of the pyramidal mirror unit equals the tilt compensation of a FSM, the aberration loss due to the tilt is completely eliminated. Therefore there is no theoretical tilt tolerance for the pyramidal mirror as long as it does not exceeds the maximum compensation range $(0.01 \mathrm{deg})$.

The beam combiner was tilted along the $x$ and $y$ axes while leaving other optical components perfectly aligned (Fig. 12). This beam combiner misalignment is not correctable by optical actuators like FSMs. However, it turns out that we can tolerate up to $0.2 \mathrm{deg}$ for the beam combiner, which is less stringent than other misalignment tolerances.

\section{Control System}

\subsection{Attitude Control System Overview}

The final ARGOS structure and the major ACS components are depicted in Fig. 1. The FOV of the main CCD is 3 arcmin and thus to give us $1 / 2$ arcmin of margin on either side, the ACS subsystem is required to provide a pointing accuracy of \pm 1 arcmin. The period of operation of the ARGOS system without human intervention must be 60 min or greater (see Table 1), meaning that the ACS system will have to either not saturate its actuators, or have some way of desaturating them within this given time span. The sys-
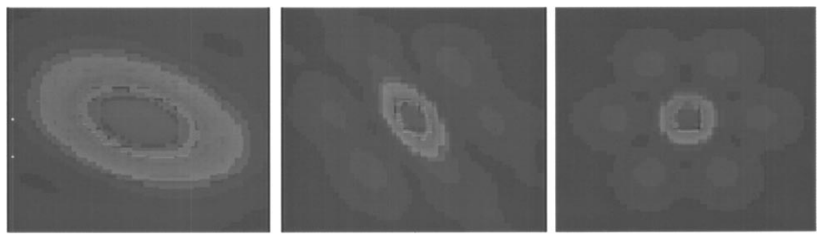

Fig. 12 PSF plots when the beam combiner has tilt errors. From left to right, $\mathrm{X}$ tilt: $0.2, \mathrm{Y}$ tilt: $0.4 ; \mathrm{X}: 0.3 \mathrm{Y}: 0.3 ; \mathrm{X}: 0.25 \mathrm{Y}: 0.25$ (deg).

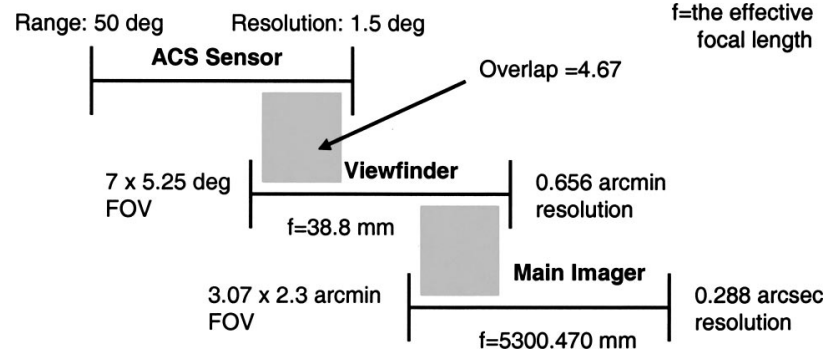

Fig. 13 Multistaged ACS sensors.

tem must be able to slew at a rate of at least $1.5 \mathrm{deg} / \mathrm{s}$, placing a minimum requirement on the capabilities of the reaction wheels to slew the spacecraft. Due to the nature of the air bearing system chosen to simulate the space-based operation of ARGOS, the center of gravity and the center of rotation of the body will not necessarily be at the same position. Three perpendicular precision linear slides of the active balancing system ${ }^{12}$ (ABS) correct for small center of mass offsets that would hurt the closed-loop performance. The ABS can also be used to remove momentum from the wheels by intentionally causing an offset in the appropriate direction (see Fig. 1).

The sensor suite is composed of three integral elements. First, the TCM-2-50 electronic compass, with a three-axis magnetometer, a two-axis tilt sensor, and facilities to provide temperature information. Inclinometers/electronic compasses measure the relative angles between the inertial coordinate frame and the body fixed frame. That is, they are used to give relative elevation, roll, and azimuth information between these two coordinate systems. The inclinometer/electronic compass is an essential component of the coarse pointing sensor suite. Second, there is an intermediary sensor, which takes the form of a scope. It is the intermediate wide FOV CCD that provides sufficient overlap (greater than 4) between the TCM (Fig. 13) and the main imager. Third, there is a three-axis rate gyroscope for angular rate measurement.

\subsection{Control Hardware and Avionics}

The HEPC8 digital signal processing module houses a TI C6701 digital signal processor (DSP), two 12-bit eightchannel digital-to-analog (D/A) (three signals for each FSM and three RWA) and one 14-bit eight-channel analog to digital (A/D). Each reaction wheel speed command from the DSP is sent through smoothing filters with a cutoff frequency of $280 \mathrm{~Hz}$. The internal analog motor controller employs a proportional integral derivative (PID) logic using the motor encoder sensor outputs. A set of three piezoelectric stacks for a single aperture FSM (a total of nine piezolinear stacks) is driven by the D/A signals, which are filtered by a $303-\mathrm{Hz}$ cutoff frequency low-pass filter and then amplified by the compact voltage amplifier (PI E0669.OE) located in the right side of ARGOS. The rate gyroscope measures the rotational rates of ARGOS and feedbacks the voltages to the A/D with a resolution of $50 \mathrm{mV} /(\mathrm{deg} / \mathrm{s})$. An antialiasing low-pass filter with a $48-\mathrm{Hz}$ cutoff frequency was implemented to reduce the highfrequency noise sent to the A/D channels. The electronic compass is directly connected to the PC motherboard using 

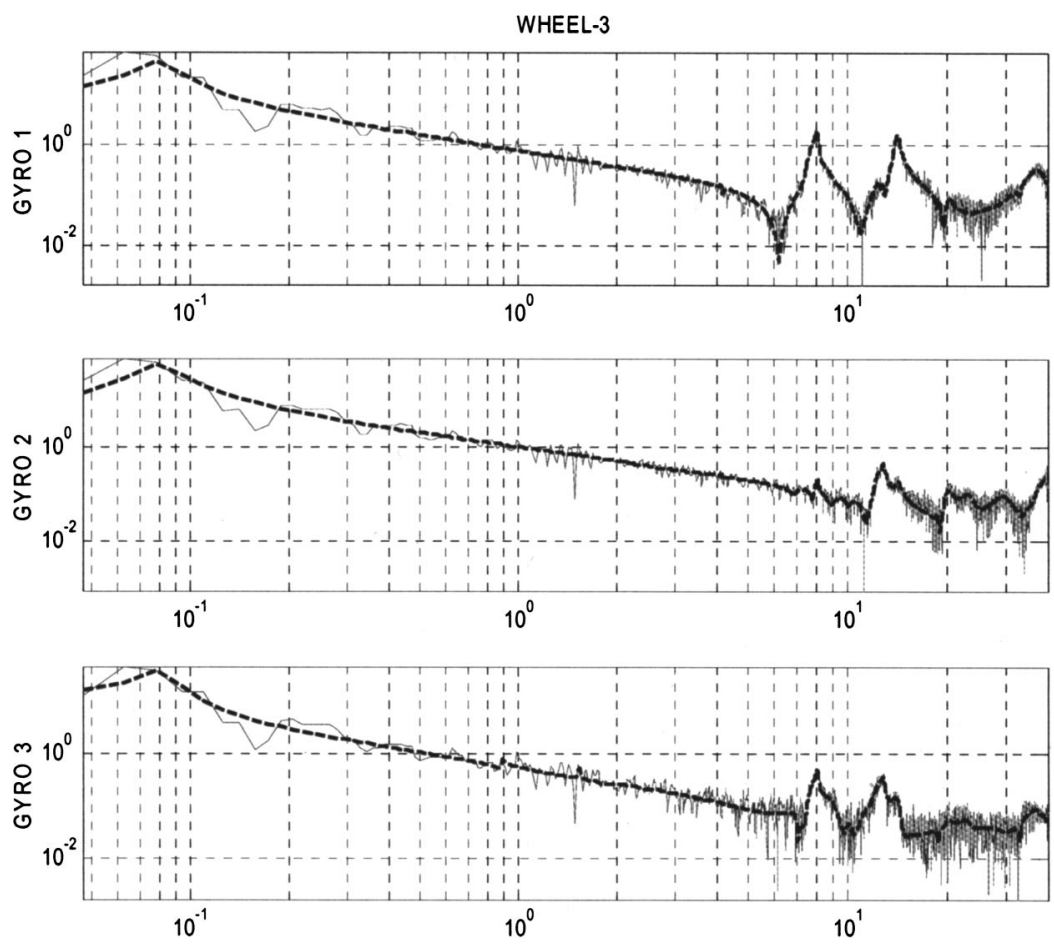

Fig. 14 System identification of ARGOS-R/W 3 to gyros 1, 2, and 3 outputs (frequency in hertz).

a standard RS232 serial port. The PC-DSP first in first out (FIFO) designated for direct communication between the host PC memory and the DSP memory, sends the image processing data ${ }^{12}$ such as the centroids of the target from the intermediate $\mathrm{CCD}$, and the compass readings to the DSP controller in real time. To meet the requirement of simultaneous automatic control of various subsystems, the DSP modules, the ABS controller and the wireless card for the communication with a remote laptop control ground station, all reside in the peripheral component interconnection (PCI) slots of the Athlon 1.4-GHz PC system with 1024 MB RAM. The graphic user interface (GUI) PC control application sends the reference slew speed and pointing (azimuth and elevation) for a specific target to the DSP while performing real-time image processing from both the intermediate ACS CCD and the main imager (spot-based wavefront sensing ${ }^{12}$ ).

\subsection{Pointing and Tracking Control Design}

The objective of system identification is to determine the dynamic properties of ARGOS and correctly design an appropriate control system to capture all present issues that would deteriorate the performance. The $3 \times 3$ transfer function matrix between the three D/A signals from the DSP and the three gyro outputs to the A/D port for the reaction wheel assembly (RWA) control is obtained. In this case, the system identification will give us a transfer function with the ACS filter board reference command as the input, the smoothing low-pass filter, motor controller, reaction wheels, structure, rate gyros, and antialiasing low-pass filter as the plant, and the filtered gyro signal from the ACS filter board as the output.

The transfer function of the plant is then found by dividing the cross spectral density of the input and output by the power spectral density of the input. Then, the measurement-based system identification algorithm called integrated frequency domain observability range space extraction and Least Square parameter estimation algorithm $^{10,18}$ (IFORSELS), is performed to derive a statespace representation of the system dynamics. IFORSELS algorithm integrates the frequency domain observability range space extraction (FORSE) identification algorithm, the balanced realization (BR) model reduction algorithm, and the logarithmic and additive least squares (LLS) modal parameter estimation algorithms for low-order highly accurate model identification.

The FORSE algorithm is a slight variation of the subspace identification algorithm derived by De Moor et al. ${ }^{19}$ and its objective is to minimize the following cost to obtain the system matrices, $A, B, C, D$,

$\left.J=\sum_{k=1}^{K} \| \hat{G}\left(\omega_{k}\right)-\left[C\left(\exp \left(j \omega_{k} \Delta t\right) I-A\right)\right]^{-1} B+D\right) \|_{2}^{2}$,

where $\hat{G}\left(\omega_{k}\right)$ is the frequency response samples from experiments.

The BR model reduction ${ }^{20}$ algorithm transforms a state space model to a balanced coordinate and reduces the model by deleting the states associated with the smallest balanced singular values. The LLS estimation algorithms improve the fitting of reduced models to experimental data by updating state space parameters in modal coordinates.

From the experimental transfer function measurements, a 0.01 - to $40-\mathrm{Hz}$ chirp signal generated the sampled transfer functions in Fig. 14 (solid line). Using the FORSE algorithm, an 80th-order state-space model was selected to approximate the experimental data and then BR and LLS al- 
gorithms were used to get a 60th-order IFORSELS model (shown by dotted lines in Fig. 14). The low-frequency peaks below $0.1 \mathrm{~Hz}$ is the pendulum mode of ARGOS while the first lightly damped structural mode occurs at 8 to $9 \mathrm{~Hz}$. Based on the linear model obtained, the pointing and tracking controller was successfully designed and implemented performing a large angle slew maneuver. ${ }^{21}$ The controller calculates the error quaternions ${ }^{22}$ from the rate gyroscope, the electronic compass/inclinometer and the viewfinder centroiding CCD mixing the mechanical sensors with the optical sensor (the viewfinder CCD in Figs. 1 and 13).

\section{Conclusion}

The ARGOS is successfully integrated into the full structure ready to operate. The ARGOS testbed is the first sparse-aperture array simulating a space-borne observatory in a $1-g$ environment representing real-world problems such as the vibrational coupling between a spacecraft structure and the wavefront errors propagating through the whole system. In this paper, the physical nature of interferometric arrays was studied and the design trades to meet the performance requirements were explored. A preliminary assessment showed that the beam combining problem is the most challenging aspect of sparse optical arrays. The need for optical control is paramount due to tight beam combining error tolerances. The attitude controller design in this paper is focused on the attitude control system incorporating the coarse optical sensor and general ACS sensor suite. The interferometric beam-combining controller utilizing model-based control and neural network is under development as well as software-based wavefront sensing techniques. The wavefront sensing/control requirements appear to be a major technology and cost driver. We anticipate that ARGOS will provide an experimental platform for the development of many phasing and vibration control techniques for future interferometers. Follow-on work will focus on actual versus expected imaging performance for a range of targets.

\section{Acknowledgments}

This research was supported by the National Reconnaissance Office (NRO) Director's Innovation Initiative (DII) under contracts number NRO-000-01-C-0207. The authors gratefully acknowledge the contributions of Dr. Carl Blaurock at Midé Technology Corporation and Dr. Alice K. Liu at the NASA Goddard Space Flight Center.

\section{References}

1. A. B. Meinel, "An overview of the technological possibilities of future telescopes," in Proc. ESO Conf. on Optical Telescopes of the Future, F. Pacin i, W. Richter, and R. N. Wilson, Eds., pp. 13-26 ESO, Geneva (1978)

2. P. R. Lawson et al., Principles of Long Baseline Stellar Interferometry, Course Notes from the 1999 Michelson Summer School, P. R. Lawson, Ed., NASA Jet Propulsion Laboratory (2000).

3. M. Golay, "Point arrays having compact non-redundant autocorrelations," J. Opt. Soc. Am. 61, 272-273 (1971).

4. J. E. Harvey, P. R. Silverglate, and A. B. Wissinger, "Optical performance of synthetic aperture telescope configurations," Proc. SPIE 540, 110-118 (1985)

5. M. Faucherre, F. Merkle, and F. Vakili, "Beam combination in aperture synthesis from space: field of view limitations and $(\mathrm{u}, \mathrm{v})$ plane coverage optimization," in New Technologies for Astronomy, Proc. SPIE 1130, $138-145$ (1989).
6. A. B. Meinel and M. P. Meinel, "Large sparse-aperture space optical systems," Opt. Eng. 41(8), 1983-1994 (2002).

7. C. R. De Hainaut et al., "Wide field performance of a phased array telescope," Opt. Eng. 34(3), 876-880 (1995).

8. V. Zarifis et al., "The multi aperture imaging array," in ASP Conf. Series 194, Working on the Fringe: Optical and IR Interferometry from Ground and Space, Unwin and Stachnik, Eds., pp. 278 (1999).

9. D. C. Redding et al., "Wavefront sensing and control for a NextGeneration Space Telescope," Proc. SPIE 3356, p. 758-772 (1998).

10. D. W. Miller et al., "The middeck active control experiment (MACE): summary report," Space Engineering Center, MIT, SERC\#7-96 (1996).

11. G. J. W. Mallory, A. Saenz-Otero, and D. W. Miller, "Origins testbed: capturing the dynamics and control of future space-based telescopes," Opt. Eng. 39(6), 1665-1676 (2000).

12. S.-J. Chung, "Design, implementation and control of a sparse aperture imaging satellite," MS Thesis, Department of Aeronautics and Astronautics, MIT (2002).

13. J. E. Harvey and R. A. Rockwell, "Performance characteristics of phased array and thinned aperture optical telescopes," Opt. Eng. 27(9), 762-768 (1988).

14. B. Mennesson and J. M. Mariotti, "Array configurations for a space infrared nulling interferometer dedicated to the search for earthlike extrasolar planets," Icarus 128, 202-212 (1997).

15. W. J. Smith, Modern Optical Engineering, 3rd ed., McGraw-Hill, New York (2000).

16. J. R. Fienup, "MTF and integration time versus fill factor for sparseaperture imaging systems," in Imaging Technology and Telescopes, Proc. SPIE 4091A-06, 43-47 (2000).

17. J. R. Wertz and W. J. Larson, Space Mission Analysis and Design, 3rd ed., Microcosm Press, El Segundo, CA (1999).

18. K. Liu, R. N. Jacques, and D. W. Miller, "Frequency domain structural system identification by observability range space extraction," ASME J. Dyn. Syst. Meas. Control 118(2), 211-220 (1996).

19. B. De Moor et al., "A geometrical approach for the identification of state space models with singular value decomposition," in Proc., IEEE ICASSP, vol. 4, pp. 2244-2247, New York, NY (1998).

20. K. Zhou, J. C. Doyle, and K. Glover, Robust and Optimal Control, Prentice Hall, Upper Saddle River, NJ (1996).

21. S.-J. Chung, D. Lobosco, D. W. Miller, and C. Blaurock, "Multidisciplinary control of a sparse interferometric array satellite test-bed," in Proc. AIAA Guidance, Navigation and Control Conf., Austin, TX (2003).

22. J. L. Marins, X. Yun, E. R. Bachman, R. B. McGhee, and M. J. Zyda "An extended Kalman filter for quaternion-based orientation estimation using MARG sensors," in Proc. 2001 IEEE/RSJ Int. Conf. on Intelligent Robots and Systems, Maui, HI (2001).

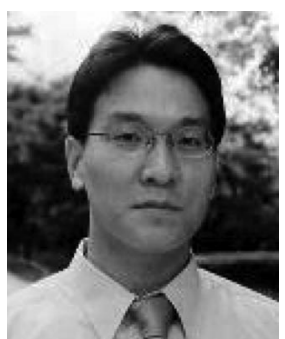

Soon-Jo Chung received his BS degree in aerospace engineering in 1998 from the Korea Advanced Institute of Science and Technology (KAIST) and his MS degree in aeronautics and astronautics in 2002 from the Massachusetts Institute of Technology (MIT), where he is currently a $\mathrm{PhD}$ candidate. During 2002, he was a research engineer for the Giant Segmented Mirror Telescope project in the National Optical Astronomical Observatories (NOAO), Tucson, Arizona. His research focuses on the control and estimation of large complex space telescopes and interferometers.

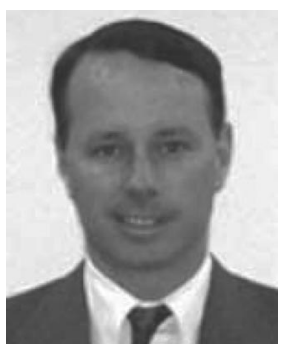

David W. Miller is an associate professor and the director of the Space Systems Laboratory in the Department of Aeronautics and Astronautics at the Massachusetts Institute of Technology (MIT). His research focus is on dynamics, controls, and systems engineering as applied to distributed satellite systems and precision optical telescopes. He has developed a series of ground testbed and Shuttle flight facilities for the conduct of research into dynamic modeling and control synthesis for precision optical systems envisioned under the National Aeronautics and Space Administration's (NASA) Origins Program. He is also working with the Air Force on system design, aperture synthesis, and formation flying for a spacebased synthetic aperture radar concept. He is a member of the NASA Terrestrial Planet Finder (TPF) science working group. 


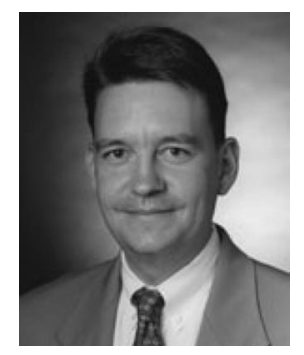

Olivier L. de Weck is the Robert N. Noyce Assistant Professor of Aeronautics Astronautics Engineering Systems at the Massachusetts Institute of Technology (MIT). His research interests are multidisciplinary design optimization (MDO) and system architecture. He earned his SM degree in 1999 and his PhD degree in 2001 from MIT and a diplomingenieur degree in industrial engineering in 1993 from ETH Zurich, Switzerland. He was the Engineering Program Manager for the Swiss F/A-18 Program at McDonnell Douglas, St. Louis, Missouri, from 1993 to 1997. 